\title{
Plasma transport modelling in the inner magnetosphere: effects of magnetic field, electric field and exospheric models
}

\author{
A. Woelfflé ${ }^{1}$, D. Boscher ${ }^{1}$, and I. Dandouras ${ }^{2,3}$ \\ ${ }^{1}$ ONERA, Toulouse, France \\ ${ }^{2}$ Université de Toulouse, Centre d'Etude Spatiale des Rayonnements, Toulouse, France \\ ${ }^{3}$ CNRS, UMR 5187, Toulouse, France
}

Received: 15 June 2010 - Revised: 30 November 2010 - Accepted: 24 January 2011 - Published: 25 February 2011

\begin{abstract}
A qualitative study is performed on plasma transport modelling in the inner magnetosphere, revealing the significance of a model use choice and its parameterization. First, we examine particle transport using comparative analysis of both magnetic and electric field models. This work reveals that the electric field plays an important role in understanding particle dynamics and the models lead to various results in terms of plasma source, energy and particle trajectory. We then concentrate particularly on proton loss assessment considering the charge exchange phenomenon. For that, models are needed to provide a neutral hydrogen density estimation. So, exospheric models were tested in light of the Dynamics Explorer 1 measurements analysed by Rairden.
\end{abstract}

Keywords. Magnetospheric physics (Electric fields; Plasma convection) - Space plasma physics (Transport processes)

\section{Introduction}

The $\boldsymbol{E} \times \boldsymbol{B}$ drift is responsible for the transport of particles earthward from the plasma sheet to the inner magnetosphere and for their energization as they move to stronger magnetic field regions while conserving their first adiabatic invariant. As one approaches the Earth, the gradient and the curvature drifts are added to this particle transport. Along their trajectories, particles are subjected to physical loss processes, such as the simple and double charge exchange phenomenon for protons, for instance. Therefore, there are several distinct components to consider when modelling plasma transport in the inner magnetosphere: source distribution in plasma sheet, magnetic and electric field models for particle transport, exospheric model for proton losses, etc.... As an inappropriate model utilization can lead to a wrong scientific analysis, the

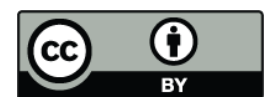

Correspondence to: D. Boscher (daniel.boscher@onera.fr) purpose of this work is to bring out how sensitive plasma transport is to both magnetic and electric field description and what are the key parameters in the geocorona modelling. Especially since magnetic field model comparison was done in the past (Walker, 1976; Spence et al., 1987; Stern, 1994; Reeves et al., 1996; Thomsen et al., 1996; Pulkkinen and Tsyganenko, 1996; Huang et al., 2008; McCollough et al., 2008). More recently Pierrard et al. (2008) did an interesting electric field model comparison, on plasmapause position during geomagnetic storms.

Modern physical electric field models for the inner magnetosphere use a kinetic approach. Among these models, we can find the Rice Convection Model (Harel et al., 1981) that considers the particles like multiple fluids, includes the coupling to the ionosphere and describes adiabatically drifting isotropic particle distributions with a specified magnetic field and a self-consistently computed electric field. There is also the Ring current-Atmospheric interactions Model (Fok et al., 1995) that focuses on the coupling between ring current ions and the plasmasphere: it solves the temporal evolution of the ring current ion phase space density, considering drift motion and losses due to charge exchange with exospheric hydrogen neutral atoms and Coulomb interactions with the plasmasphere. The Comprehensive Ring Current Model (Fok et al., 2001) is actually a combination of the Rice Convection Model and the Fok ring current model (Fok and Moore, 1997) where this last one plays the role of a particle tracer using the electric field computed by the RCM. The updated plasma distribution given by the Fok model is then returned to the RCM. Thus, the CRCM represents a ring current model in a self-consistently computed electric field. In spite of the recent interesting results given by kinetic models, in this paper we deliberately choose to focus on physically oversimplified electric field models with few input parameters: this allows us to introduce the less possible bias in the comparison analysis and moreover, it makes sense for a study at $\mathrm{Kp}=3$, given the fact that in plasma transport modelling

Published by Copernicus Publications on behalf of the European Geosciences Union. 
few differences are observed during quiet times between a kinetic model and a Volland-Stern analytic one, for example (Fok et al., 2001).

Here, electron and proton transport is simply encoded by a 4th order Runge Kutta integration method, assuming the conservation of the first adiabatic invariant. Usually we simulate particle transport back in time, therefore, we need not know the geometry source distribution. Calculations made by Tsyganenko and Mukai (2003) allow us to be aware of the fact that proton plasma sheet density and temperature are not homogeneous at $10 R_{\mathrm{E}}$ on the nightside along a width of $20 R_{\mathrm{E}}$ in the equatorial plane, particularly when the magnetic activity corresponds to a Dst index lower than $-20 \mathrm{nT}$. In this last case, the maximum density and temperature are obtained as a width of $3 R_{\mathrm{E}}$ on both sides of the central plasma sheet at this distance. So it highlights the need to take a realistic particle distribution when the simulation begins at the source location in the plasma sheet. Furthermore, ionic and electronic temperatures are not the same (Christon et al., 1991), so the distribution profile should also fit the particle type.

The equatorial back-in-time particle trajectories are plotted here in a system of coordinates such as $X=-X_{\mathrm{GSM}}$ or SM and $Y=-Y_{\mathrm{GSM}}$ or SM, so that we can have the Sun on the left side of the picture: note that the original system of coordinates GSM or SM of the field models is specified in the corresponding description sections.

In this paper, we carry out a qualitative comparative study of magnetic field, electric field and exospheric models in order to know which differences we get when we choose a model instead of another.

\section{Influence of magnetic and electric field models on sim- ulating plasma transport in the inner magnetosphere}

\subsection{A comparison of magnetic field models}

\subsubsection{Model description}

The magnetic environment of the Earth has been measured almost continuously during several solar cycles making it possible to develop several empirical magnetic field models, giving an average configuration according to magnetic activity (Mead and Fairfield, 1975; Olson and Pfitzer, 1977; Tsyganenko and Usmanov, 1982; Tsyganenko, 1987, 1989, 1996, 2002a, b; Pfitzer et al., 1988; Ostapenko and Maltsev, 1997; Alexeev et al., 2000; Tsyganenko et al., 2003; Tsyganenko and Sitnov, 2005). As none of these models are strictly accurate but only gives the global influence of magnetic activity on magnetic field configuration, we have selected those with very few parameters so that we may introduce the less possible bias for model comparison. We respectively study the morphology of particle trajectories, the Alfvén layer description for each field model and then, the source location as well as the temporal evolution of the particle energy, in order to know what differences each field implies on particle dynamics.

One of the earliest models is the Mead and Fairfield (1975) model which only takes $\mathrm{Kp}$ index as input. The model resolves a second-order power series in the solar magnetic coordinates to deduce the external field. It takes into account the dipole tilt and there are four datasets of coefficients corresponding to four different levels of magnetic activity. These coefficients are determined thanks to the least square method from 12616 vector measurements of the field between 1966 and 1972. The model is valid as far as 17 Earth radii.

The particularity of the Olson and Pfitzer (1977) quiet model is that it does not take any parameter as input except the tilt (and obviously the location), so basically it is more dedicated to quiet time utilization. This model uses a sixthorder power series expansion and exponential terms in position and tilt. Whatever dipole tilt value can be chosen and magnetospheric and magnetopause current contributions are included. This external field diverges quickly from 15 Earth radii so the model should not be used above this limit. Lower than $2 R_{\mathrm{E}}$, the external field is fixed to zero knowing the predominance of the internal field in this region.

The Tsyganenko (1989) model gives the magnetic field components in geocentric solar magnetic coordinates as far as 70 Earth radii in the nightside with $\mathrm{Kp}$ index as a sole input. It is built on satellite datasets (IMP, HEOS and ISEE) and incorporates the terms expressing spatial variation of the current sheet, the ring current and the magnetotail warping (linked to the dipole tilt). There are seven datasets of coefficients determined thanks to the least square method, each set corresponding to a different level of magnetic activity.

\subsubsection{Comparison analysis}

We have chosen the following spatial and particle energy conditions as input of our simulation: six protons with, respectively, 1, 2, 4, 8, 16 and $32 \mathrm{keV}$ energy arriving at the point $(-6 ; 1)$ in our system of coordinates. At the beginning, these conditions have been inspired by both Cluster and Double Star satellite observations of "nose-like" ion structures (Dandouras et al., 2009), more precisely, by the narrow ion energy bands lower than $30 \mathrm{keV}$ seen on 3 April 2004 at around 11:30 LT (i.e., around the point $(-6,1)$ ). This configuration has also been kept later for electron simulation.

As the simulation goes back-in-time, we can evaluate where these particles come from, with the three magnetic field models previously described, and compare the different particle trajectory morphologies we obtained. A 24-h period is simulated: trajectory results in the equatorial plane are illustrated in Fig. 1 where particle energy is colour-coded. A dipole is used for the internal magnetic field, a VollandStern model for the electric field modelling (more specifically Maynard and Chen, 1975 model) and the magnetic activity is chosen closed to the mean activity in the magnetosphere $(\mathrm{Kp}=3)$. The fact that the Olson and Pfitzer quiet 

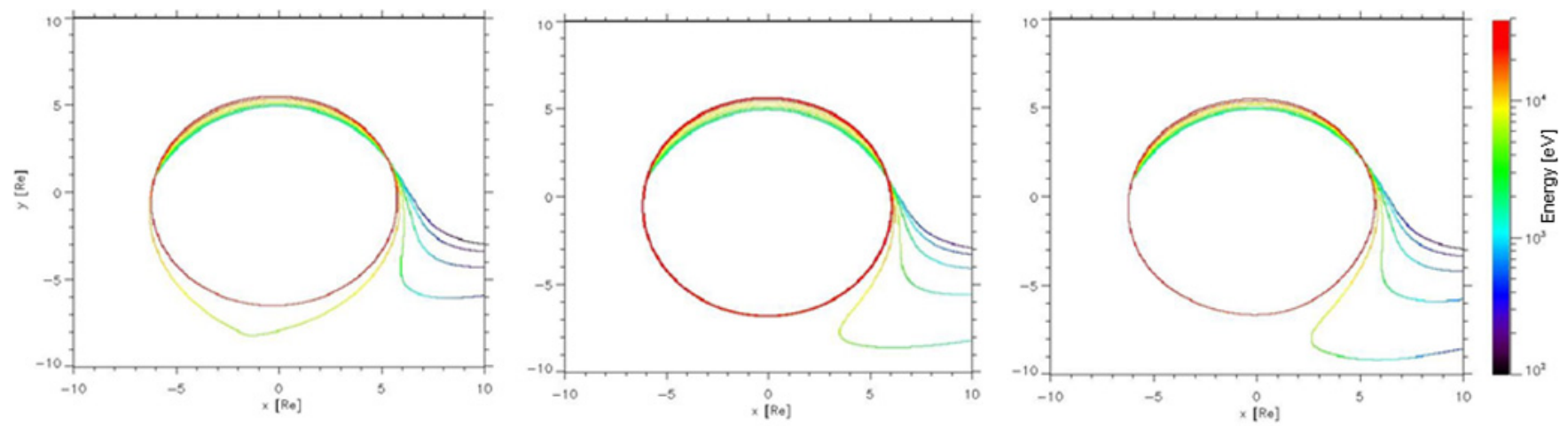

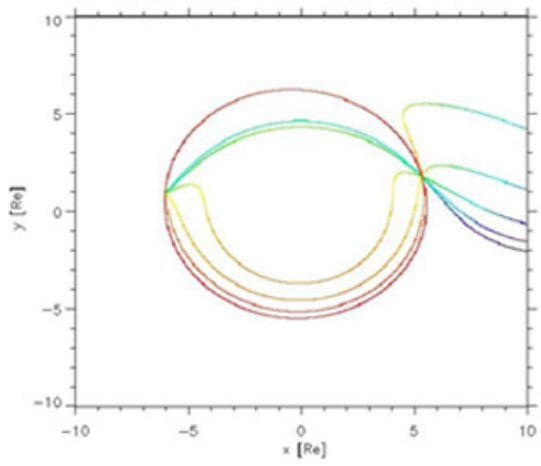

Mead \& Fairfield

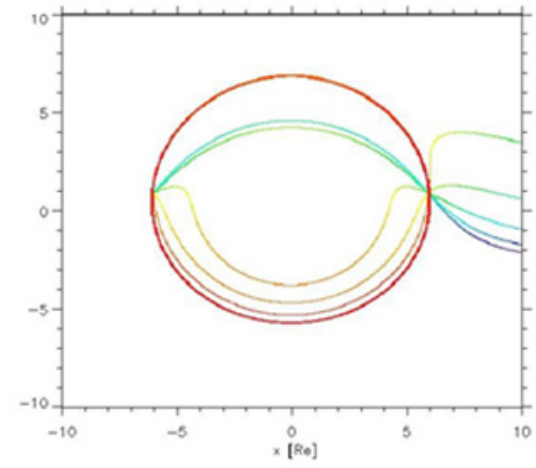

Tsyganenko 1989

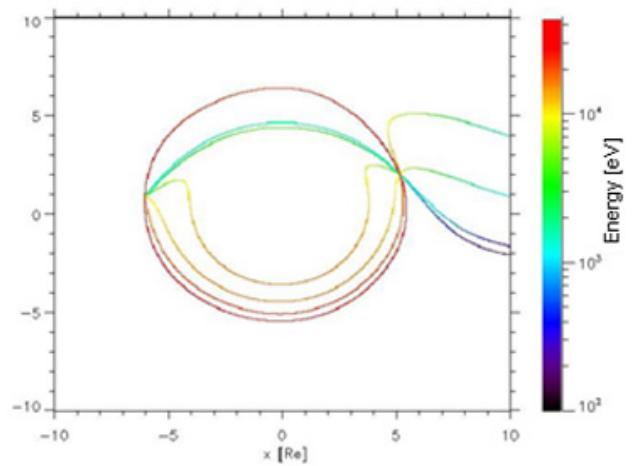

Olson \& Pfitzer quiet

Fig. 1. Electron (top panel) and proton (bottom panel) trajectories back-in-time from the point $(-6,1)$ in the equatorial plane with Mead and Fairfield, Tsyganenko (1989), and Olson and Pfitzer quiet models at $\mathrm{Kp}=3$.

model is only fit to quiet times and the fact that basic magnetic field models are not so reliable at higher values of $\mathrm{Kp}$ index, according to a McCollough et al. (2008) study, justify this choice. No obvious differences between the various models can be seen. Concerning electrons, the bigger difference may concern the particle with $16 \mathrm{keV}$ energy which is on a closed drift with the Mead and Fairfield model, contrary to the other models, but a small variation on the source localisation (or small variation on Y-axis at $X=10 R_{\mathrm{E}}$ on the nightside) can result in a switch between open and closed trajectories, so it is not so meaningful. In the proton case, particles also come from the same region of space, the only difference concerns the proton with $4 \mathrm{keV}$ energy with Olson and Pfitzer quiet model, which visibly moves from plasma sheet at $X=10 R_{\mathrm{E}}$ to the arrival point in more than $24 \mathrm{~h}$.

On the whole, as no distinction between these models is possible, we study further the Alfvén layer so that we can determine, by comparison, the influence of each model on particle dynamics. The limit between an eastward and a westward drift, given by the Alfvén layer, is found within a $\pm 0.05 R_{\mathrm{E}}$ precision on the source position (supposed here at $X=10 R_{\mathrm{E}}$ on the nightside), for both electrons and protons whose magnetic moment is $\mu=2 \times 10^{10} \mathrm{eV} / \mathrm{T}$. As in the previous study, magnetic activity is fixed at a level given by $\mathrm{Kp}=3$, a dipole is used to model Earth's intrinsic magnetic field and we keep the Maynard and Chen model for the electric field modelling. Alfvén layers are shown in Fig. 2 for each model case. The points $\mathrm{A}, \mathrm{B}, \mathrm{C}$ and $\mathrm{D}$ symbolise null-derivative points of particle trajectories: relative errors on magnetic field magnitude $\Delta \mathrm{B} / \mathrm{B}$ and radial distance $\Delta r / r$ are assessed at these locations so that we can differentiate magnetic field models. As each magnetic field model is parameterized for a Kp class, it is necessary to take a reference and estimate these same relative errors to the limits of this associated Kp interval. It is done for the Tsyganenko model by evaluating the average magnetic field magnitude and radial distance inside the Kp classes [2;3] and [3;4]. By comparing the Alfvén layers plot in these two cases and for $\mathrm{Kp}=3$, with Tsyganenko (1989) model, the worse relative errors $\Delta \mathrm{B} / \mathrm{B}$ obtained are $26.7 \%$ and $17.1 \%$, respectively, for electron and proton cases, with a $\Delta \mathrm{Kp}$ of 1 . As the magnetic field magnitude varies in $r^{-3}$, the results show that the relative errors $\Delta r / r$ obtained in model comparisons must be respectively greater than $8.9 \%$ and $5.7 \%$ to be significant. However, such values are lower than this reference in both electron and proton cases, so there is no way to appropriately compare magnetic field models here.

Then, we finally try to differentiate these three basic magnetic field models with other criteria. For that, we pick up the values of particle energy, drift time and Y-position at 

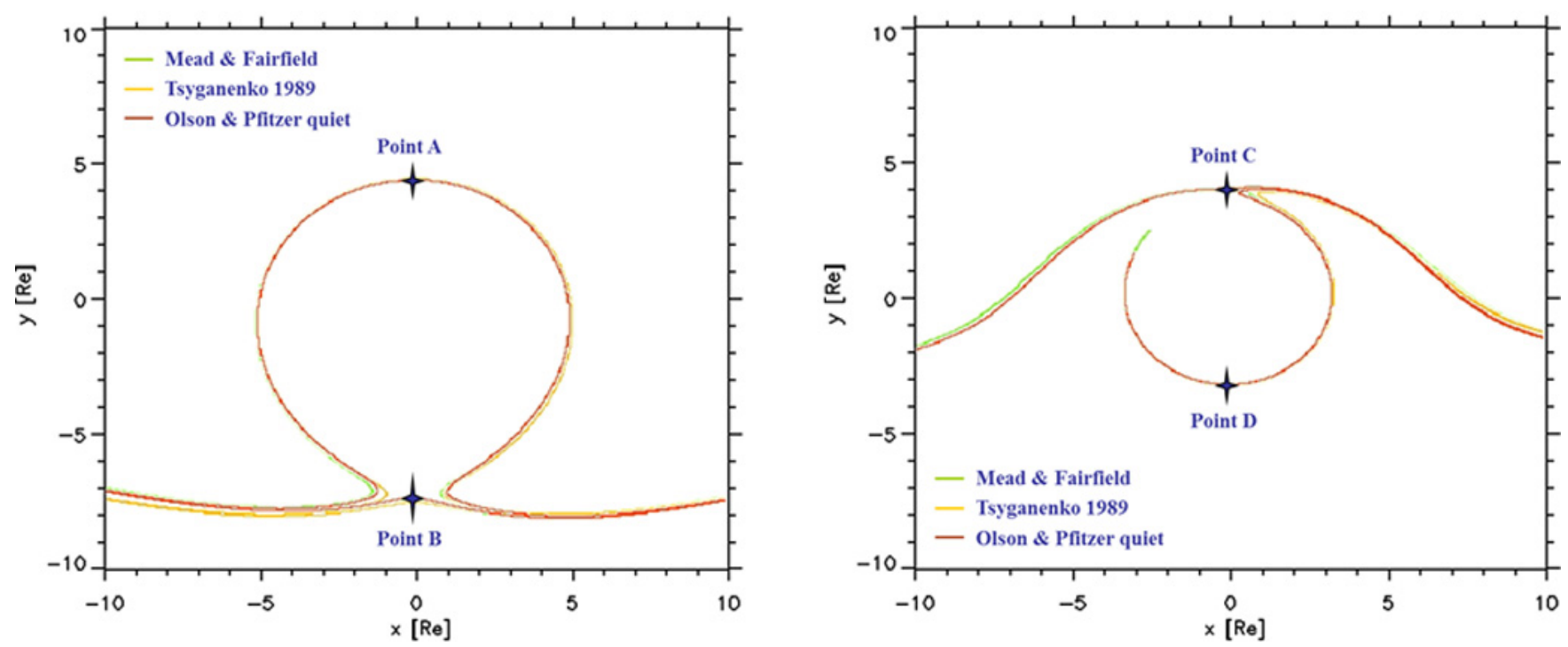

Fig. 2. Alfvén layers in the equatorial plane with Mead and Fairfield, Tsyganenko (1989) and Olson and Pfitzer quiet models for electrons (on the left) and protons (on the right) at $\mathrm{Kp}=3$.

$X=10 R_{\mathrm{E}}$ on nightside during the back-in-time simulation detailed at the beginning of this section. The Y-position criterion does not lead to distinguishing a magnetic field model from another, actually few variations on source localisation is seen $\left(\Delta Y=-0.3 / 0.5 R_{\mathrm{E}}\right.$ in, respectively, electron and proton cases, between Mead and Fairfield and Tsyganenko, 1989, models). Concerning particle drift time, the analysis shows that Olson and Pfitzer quiet model gives a mean drift time value with regards to the other models. In the electron case, the maximum drift time ratio equal to 1.17 is obtained for the 4th launched particle (with $8 \mathrm{keV}$ energy at the departure point of the simulation), by comparing Tsyganenko 1989 model (taken all the time as a reference) to the Mead and Fairfield model. In the proton case, the maximum drift time ratio is equal to 1.33 with the 2 nd particle (with $2 \mathrm{keV}$ energy at the simulation beginning) and is obtained for the comparison between the Tsyganenko (1989) and Olson and Pfitzer quiet models. These critical values are obtained near the eastward/westward drift transition. Particle energy is the criterion which leads to the most important differences: globally, particle energy variation between magnetic field models becomes stronger when the energy we consider is greater. In the electron case, the maximum energy ratio reaches 3.78 and is obtained with the 5th launched particle (with $16 \mathrm{keV}$ energy at the departure point), by comparing Tsyganenko (1989) model to Olson and Pfitzer quiet model. In the proton case, the maximum energy ratio is 3.30 and is obtained by comparing Tsyganenko (1989) model to Mead and Fairfield one, with the same previous particle. Tsyganenko (1989) model always gives the greater energy values but they remain in a realistic few $\mathrm{keV}$ interval. Besides, as this model works in GSM coordinates, it probably gives better results far from the Earth as the GSM coordinates fit better with the magnetotail orientation according to the Sun position $\left(X_{\mathrm{GSM}}\right)$ than the SM coordinates do.

Obviously, the differences between the basic Mead and Fairfield, Tsyganenko (1989) and Olson and Pfitzer quiet magnetic field models are very thin. Because of the reason mentioned above and the fact that Tsyganenko (1989) model is the basis for more recent evolved models (studied in detail by McCollough et al., 2008, and Huang et al., 2008), we will keep this model as a reference in the next electric field model comparison analysis. Moreover, although the Olson and Pfitzer quiet model gives good results at $\mathrm{Kp}=3$, taking magnetic activity into account is essential in understanding the particle dynamics in the magnetosphere, that is the reason why magnetic activity parameter(s) should be considered as input of a magnetic field model.

\subsection{A comparison of electric field models}

\subsubsection{Model description}

The morphology and dynamics of the ring current closely depend on the Inner Magnetospheric Electric Field (IMEF, cf. Wygant et al., 1998), which in turn depends on solar wind and ionosphere dynamics. The IMEF can be represented for a first order approximation by the superposition of a constant, global, cross-tail merging electric field (convection electric field) and the corotation electric field.

Among all convection electric field models, the VollandStern model (Volland, 1973; Stern, 1975) is one of the most famous, probably due to its simplicity. This model gives an analytic expression for the global, scalar electric field potential of the Earth's inner magnetosphere. In this model, no 

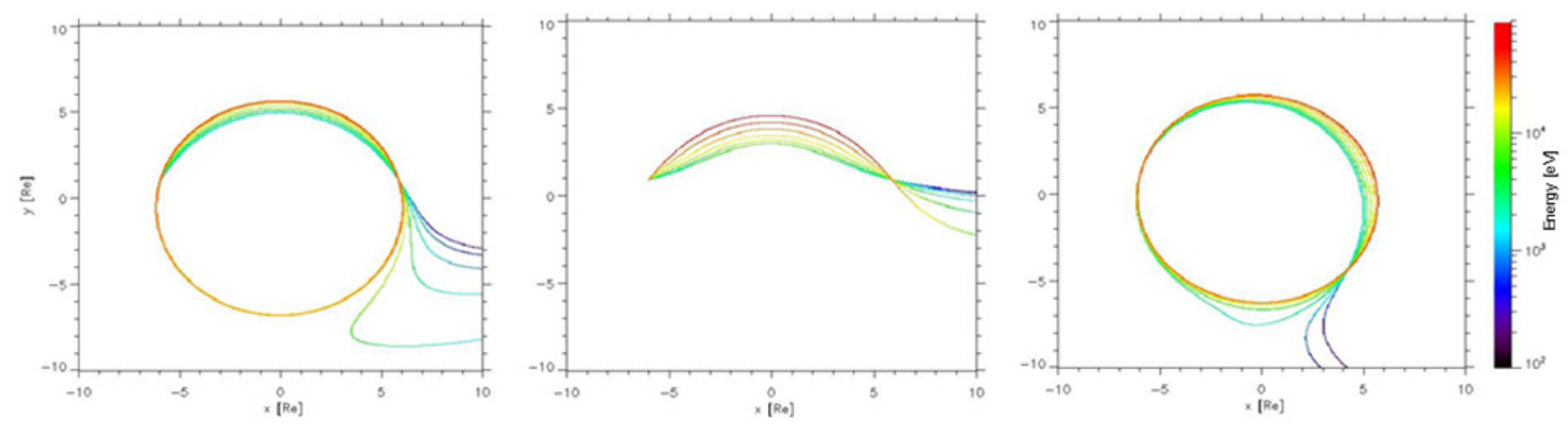

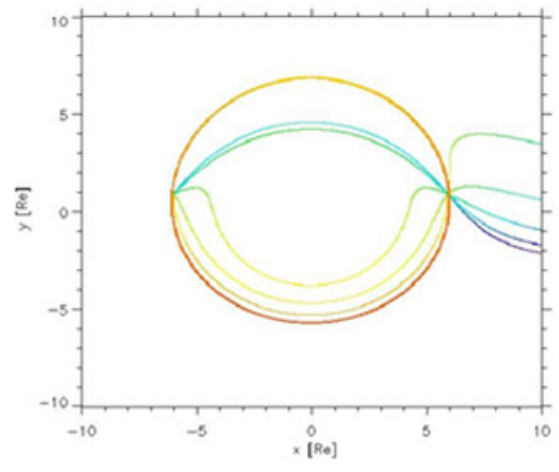

Maynard \& Chen

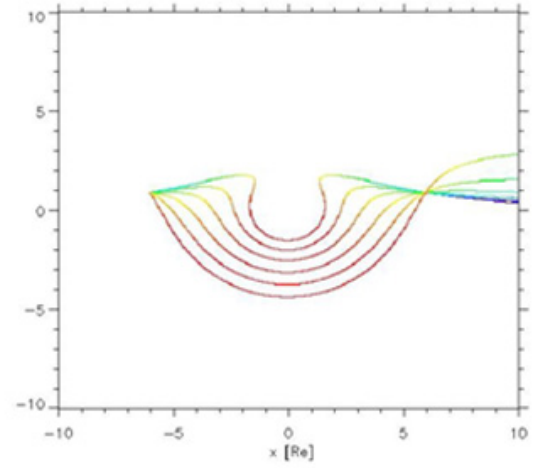

Whipple

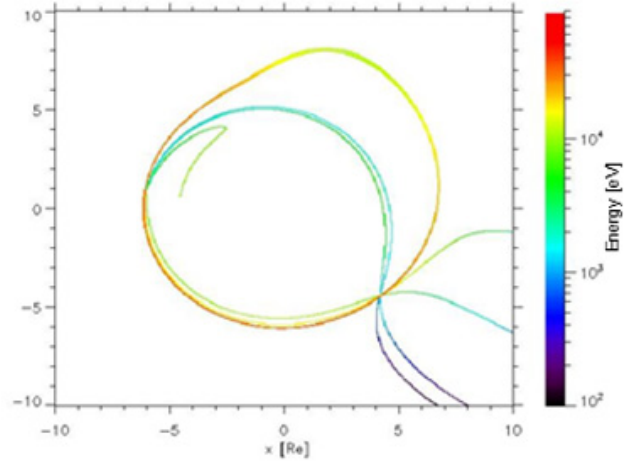

McIlwain

Fig. 3. Electron (top panel) and proton (bottom panel) trajectories back-in-time from the point $(-6,1)$ in the equatorial plane with the Maynard and Chen, Whipple and McIlwain models $\left(\mu=\left\{7.2 \times 10^{6} ; 1.4 \times 10^{7} ; 2.9 \times 10^{7} ; 5.7 \times 10^{7} ; 1.2 \times 10^{8} ; 2.3 \times 10^{8}\right\} \mathrm{keV} / \mathrm{T}\right.$ for $E_{0}=\{1 ; 2 ; 4 ; 8$; $16 ; 32\} \mathrm{keV}$ at $\mathrm{Kp}=3$ ).

induced electric field resulting from time dependent magnetic field variations is taken into account. The VollandStern electric convection potential is originally written as a function of the cross-polar cap potential by $\Phi=-A r^{\gamma} \sin \phi$, where $r$ is the equatorial distance, $\phi$ the azimuthal angle from noon, $\gamma$ the shielding factor and $A$ is a factor which determines the convection electric field intensity.

The geomagnetic activity dependence of this model, thanks to the Kp index parameter, was later revised by Maynard and Chen (1975) by adjusting the last closed equipotential of the total electric field with the position of the plasmapause observed by the satellites OGO. The factor $A$ is expressed as followed:

$$
A=\frac{0.045}{\left(1-0.159 \mathrm{Kp}+0.0093 \mathrm{Kp}^{2}\right)^{3}}\left[\mathrm{kV} \mathrm{Re}^{-2}\right]
$$

and in the total electric field expression, the shielding parameter $\gamma$ is equal to two and the coefficient $B$ in the corotation term $-B / r$ to $92.4 \mathrm{kV}$ Re.

When the shielding parameter $\gamma$ is taken as equal to one, the electric field is unshielded: this is the case of the electric field expression used by Whipple Jr. (1978) in his study about magnetospheric plasma convection. The factor $A$ was taken equal to $10 \mathrm{kV} R_{\mathrm{E}}^{-1}$ and the corotation coefficient $B$ to $92 \mathrm{kV} R_{\mathrm{E}}^{-1}$. This is what we will call "Whipple model" in the next section.

Nevertheless, various authors choose to use a VollandStern model with $\gamma \approx 2$ (Korth et al., 1999) where the shielding is proportional to the radial distance. More recently, Ebihara and Ejiri (2000) have even referred the convection electric field directly to the solar wind parameters.

The E5D model derived by McIlwain (1986) is another analytical representation of the magnetospheric electric field, which was constructed using electron and proton measurements from the ATS-5 and ATS-6 satellites at a geosynchronous orbit. In this model, the convection potential depends on a shielding function $H$, very different from Volland-Stern shielding, which also depends on magnetic activity by the $\mathrm{Kp}$ index. For instance, at midnight and for $\mathrm{Kp}=6$, the shielding is efficient $(H=1 / 2)$ for a radial distance around $3 R_{\mathrm{E}}$. Globally, the shape of the function $H$ shows that above this distance shielding is not effective, while below it is nearly complete. Besides, McIlwain's equipotential lines are orientated around a symmetry axis rotated clockwise to one hour from the VollandStern dawn-dusk symmetry. This model can well predict the 

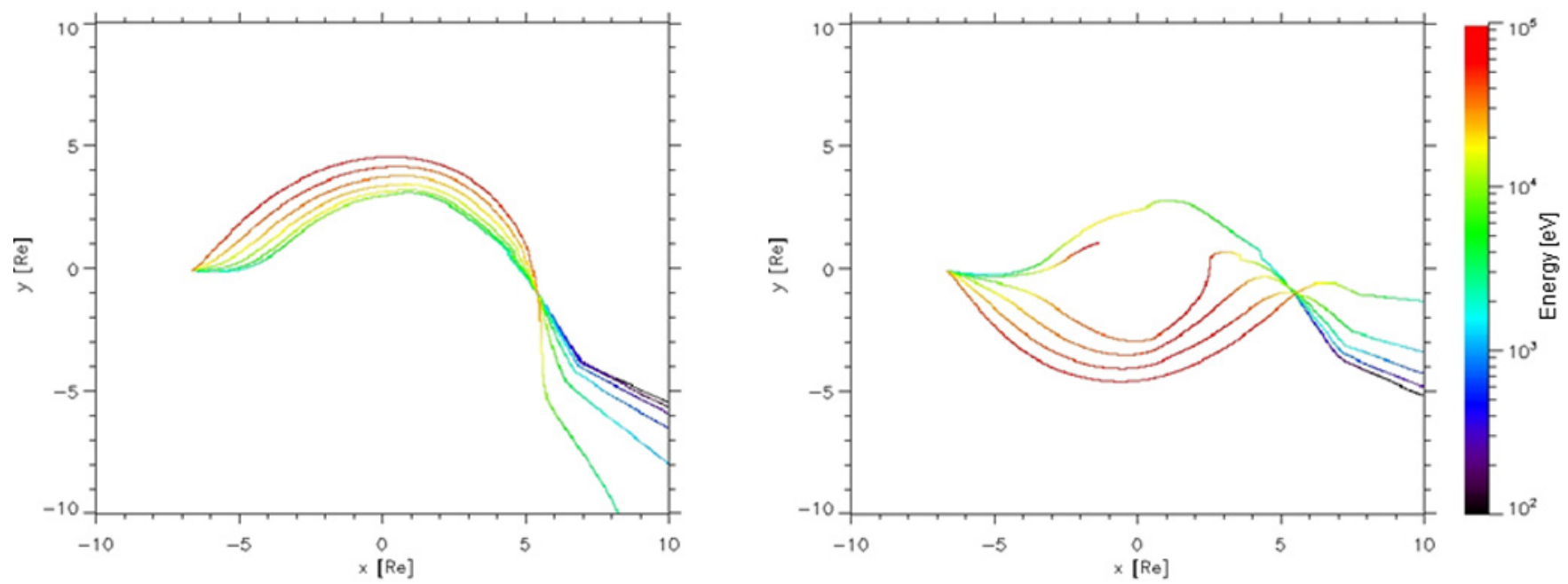

Fig. 4. Electron and proton trajectories back-in-time from the point $(-6,1)$ in the equatorial plane for a medium magnetic activity $(\mathrm{Kp}=6)$ with Liemohn electric field potential (respectively on the left and right sides).

plasmapause position according to the Pierrard et al. (2008) study. It must be noted that the Kp dependence of the shielding function was modified by Liemohn et al. (2001).

\subsubsection{Comparison analysis}

The same kind of simulation is done here: again we consider six particles in the equatorial plane, both protons and electrons, respectively with $1,2,4,8,16$ and $32 \mathrm{keV}$ energy when they arrive at the final point $(-6 ; 1)$ in our back-in-time run. A dipole and Tsyganenko (1989) model are used for the magnetic field modelling and the magnetic activity is fixed to $\mathrm{Kp}=3$. The trajectory results are illustrated on Fig. 3 with the Maynard and Chen, Whipple and Mcllwain models. We have noticed that electric field models have a great influence on particle dynamics inside the magnetosphere: this trajectory visualisation with different models leads us to think that convection electric field is not really well-known today and its understanding is crucial for a better comprehension of the inner magnetosphere physics. What is interesting is that these models get a very different shielding: from none for the Whipple model to a complex one with the McIlwain model, so electric field shielding appears to be a key point to let us know where particles come from, how they reach the nearEarth region and what occurs when particles are injected during geomagnetic storms. With the Whipple model, because of the absence of shielding, the particles can approach very close to the Earth, this is the reason why they gain much more energy (few tens of $\mathrm{keV}$ in this example) when they drift around the Earth, contrary to other field models. In 2002, Ridley and Liemohn deduced an electric field potential from a storm time asymmetric ring current model. Potential values were generated from subauroral field-aligned currents out of the ionosphere in the midnight sector and into the ionosphere on the dayside. A computation of this electric potential in given conditions at $\mathrm{Kp}=6$ (Liemohn, 2008) results in a similar trajectory distribution in the inner magnetosphere (see Fig. 4). So it may be possible that the Whipple model is better fit for higher magnetic activity levels when particles are able to penetrate deeper earthward. The McIlwain model is far from this configuration: firstly, particles drift farther from the Earth, due to a strong and complex shielding and secondly, low-energy particles seem to come from a surprising location. Actually, it appears that they issue from the 18:00 21:00 LT sector, especially in the electron case, so relatively far from the plasma sheet geometry (Tsyganenko and Mukai, 2003). However, there are still some particles coming from plasma sheet (for some higher energy protons). In the Maynard and Chen model, we find that higher energy particles are trapped like in the previous case and we observe around the same distance from Earth to particle drift paths. Nevertheless, the particle origin is closer to the Whipple estimation: the source globally expands on both sides of the central plasma sheet, inside the 21:00-03:00 LT sector.

To better understand where the particle source is localised with the Mcllwain model, the same kind of simulation as before was done, except we ran the simulation for each hour in local time, so 24 runs in total, at the geostationary orbit distance and without the external magnetic field model (only a dipole). Superposing the results in both electron and proton cases allows us to be aware of the source extent with this model. Actually, protons seem to come from the whole 21:00-03:00 LT sector, whereas electrons only come from the dusk-midnight region. In the proton case, there is a split in energy: for low-energy protons up to $1 \mathrm{keV}$, we find the same source location as in the electron case, which can be explained by the fact that low-energy protons are drifting like electrons; and over this value, protons originate from 
a large plasma sheet region (almost $20 R_{\mathrm{E}}$ wide). For electrons, particles which come from the closer region to the central plasma sheet are obtained for a simulated arrival point localised at 02:00-03:00 LT: this is consistent with Korth et al. (1999) observation of an electron flux maximum at these local times. If electrons really come from the central plasma sheet where the particle density is stronger, and on a direct path, where the drift time is shorter, then it should explain this maximum flux.

An electric potential comparison at the particle local time arrival versus the radial distance was also done for the three electric field models. Up to $2 R_{\mathrm{E}}$, electric potential values are almost equivalent. Over one Earth radius of altitude, deviation is getting more and more important between models. The strongest potential differences we get, with the Maynard and Chen model taken as a reference, are $2.7 \mathrm{kV}$ at $r=7 R_{\mathrm{E}}$ with the McIlwain model and $14 \mathrm{kV}$ at $r=10 R_{\mathrm{E}}$ with the Whipple model. The Whipple electric potential is getting greater and greater compared to the other model values when radial distance increases.

It is interesting also to note that particle drift paths, shown in Fig. 1 and Fig. 3, exhibit a kind of conjugate point of the arrival point $(-6,1)$, where all the particle trajectories intersect, whatever the particle energy and its charge are. This characteristic of an image position where a particle has the same energy as at its original position has already been observed by Kovrazhkin et al. (1999). By conserving the total energy, it can be trivially shown that these two points are on the same equipotential line. Then it is possible to deduce the conjugate point for a given original point according to the electric field model. As Maynard and Chen and Whipple models are both based on a Volland-Stern electric potential formulation, they show the same symmetry. Knowing that our particle arrival point and its conjugate point are on a given equipotential line, we can easily deduce a relation between the local times at each point. It then defines the local time $\mathrm{LT}=\pi / 2$ as the axis of symmetry, if we assume that the magnetic field is a simple dipole. In the McIlwain case, the expression of symmetry is nonlinear and more complex, partly due to the shielding factor equalled to 8 . To get an idea, if the conjugate point is at midnight, we can expect to have its pair at around 06:40 LT according to the simulation results, compared to noon for a Volland-Stern formulation.

A parameterized equatorial electric field model for the inner magnetosphere based on Cluster EDI and EFW data merging was recently derived by Puhl-Quinn et al. (2008), providing electric field mappings for different levels of magnetic activity. We decided to compare the Maynard and Chen and McIlwain electric field models during quiet times with this empirical model. Although the Puhl-Quinn et al. electric field mappings are parameterized by classes of the Zcomponent of the Interplanetary Magnetic Field, we computed our basic models with $\mathrm{Kp}=3$ so that we can make a comparison with the $-5<\mathrm{IMF} B_{\mathrm{Z}}<0 \mathrm{nT}$ mapping. The convection electric field magnitude is rotated clockwise by $90^{\circ}$ in order to put the vector in the convection direction: equatorial map results are illustrated in Fig. 5, in a frame corotating with the Earth in the SM coordinates. It results that the Maynard and Chen convection term is too simplistic for modelling a realistic topology of the convection electric field with regards to the Puhl-Quinn et al. mapping. In fact, the distribution is symmetrical around the noon-midnight axis, so the model can globally describe the convection electric field topology, but not accurately enough locally. Otherwise, the McIlwain convection electric field mapping seems more realistic. The topology looks similar to the Puhl-Quinn empirical one, with the important exception that the strongest convection is obtained in the midnight-dawn sector, whereas it is obtained on the opposite side with the Cluster data empirical model, i.e., in the noon-dusk sector.

By these different analyses, we have obtained an idea of the advantages and disadvantages of each basic electric field model studied here: in what type of conditions they perform well, but do not allow us to conclude which is the more appropriate model to describe the real electric field configuration in the Earth's magnetosphere.

\subsection{Summary}

Some efforts are currently done in order to get models which are both self-consistent and close to the physics. Having a better specification of the magnetic and electric fields is one of the first steps in succeeding. The comparison analysis done here with basic magnetic and electric field models leads us to the following comments:

1. No significant differences were noticed with the consecutive use of Mead and Fairfield, Tsyganenko (1989) and Olson and Pfitzer quiet models. The few input parameters of these basic magnetic field models prevented an accurate description of the magnetic field inside the magnetosphere, but the advantage is that they are very easy to use and because of their low number of input, there is a better understanding of the influence of a given physical parameter on the final field configuration. Magnetic activity should be considered as a magnetic field model input, that is the reason why the Olson and Pfitzer quiet model must not obviously be used beyond quiet time conditions.

2. Electric field configuration in the inner magnetosphere is not well understood and is a key point for the plasma transport modelling. Using various electric field models, such as the Maynard and Chen, Whipple and McIlwain models, leads us understand the electric field shielding is a crucial point. The Maynard and Chen model gives a good global description of particle transport from the plasma sheet to the Earth, but from a local point of view, it is not accurate enough. Plasma source is located inside the plasma sheet, as for the Whipple model. Source location with the McIlwain model is 

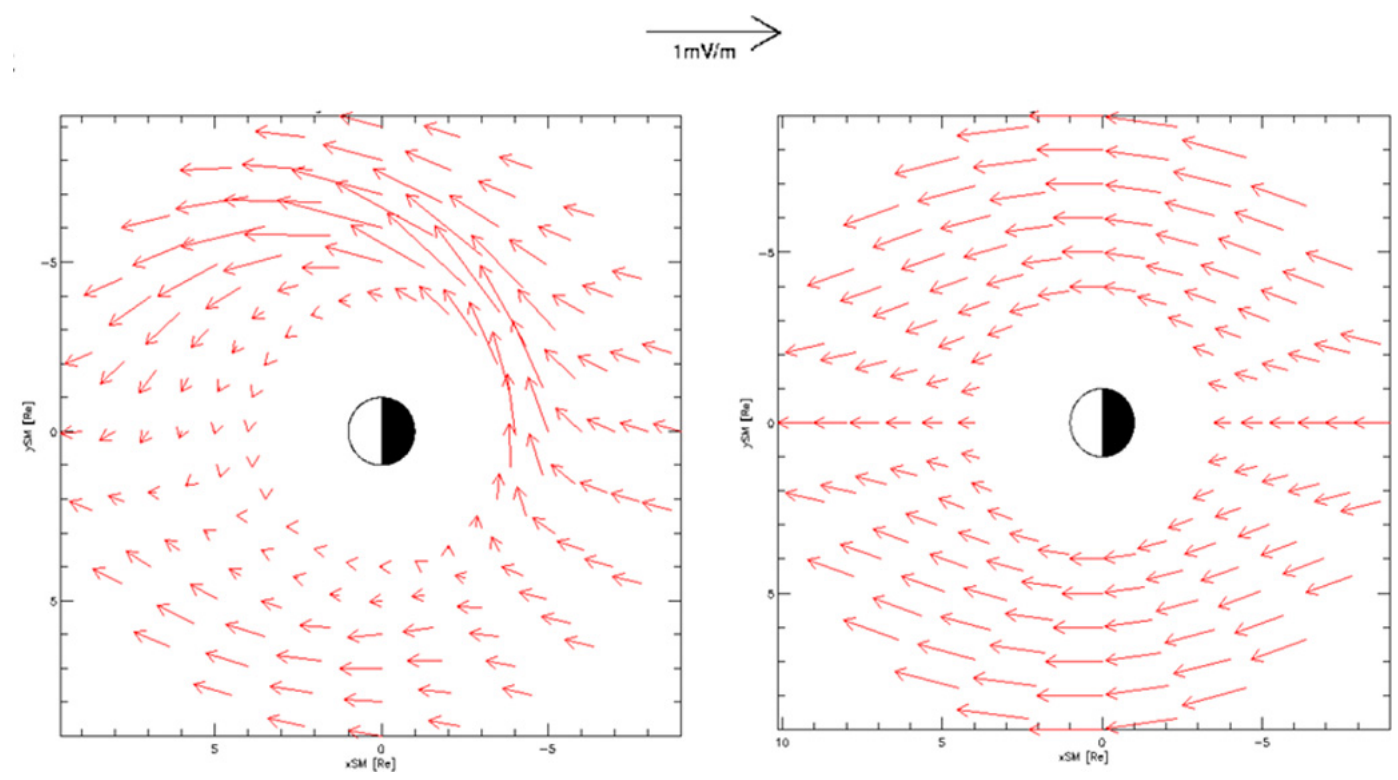

Fig. 5. Plasma convection direction with the electric field given by the McIlwain model (on the left) and the Maynard and Chen one (on the right): corotation electric field is not included in these two figures.

not so easy and sometimes low energy particles seem to come from a region outside the plasma sheet. However, the McIlwain model gives a more accurate electric field topology than the other models inside the plasmasphere. Moreover, its shielding expression is interesting because it is magnetic-activity dependent. The absence of shielding with the Whipple model prevents a fitting electric field description for quiet times, but during higher magnetic activity periods, the model gives a global view of particle penetration inside the inner magnetosphere. Note that the absence of shielding was also investigated by Thomsen et al. (2002) and Burke (2007).

Now we will focus on loss phenomena that particles undergo during their transport. Many loss processes are at stake in the magnetosphere: electrons are mainly lost by particle wave interactions, such as electromagnetic ion cyclotron waves (EMIC) in plasmaspheric plume, plasmaspheric hiss waves inside the plasmasphere, and whistler mode chorus waves outside for instance, but these phenomena are not discussed here. Concerning proton loss interactions, we can mention the Coulomb collisions (Coulomb drag) and the charge exchange. As this last phenomenon plays a prominent role in the inner magnetosphere and mainly occurs between a proton and a neutral hydrogen atom, we are interested here in estimating the exospheric hydrogen density. That is the reason why we aim at testing several exospheric models in the next section and comparing them to hydrogen density measurements.

\section{Influence of exospheric models on neutral hydrogen atom density estimation and approximated loss assessment}

\subsection{A comparison baseline}

Few data of atomic hydrogen density in the exosphere are available. The geocoronal measurements obtained by an ultraviolet photometer onboard the Dynamics Explorer 1 satellite from 1981 through 1985 were used by Rairden et al. (1986) in order to characterise the global density distribution of exospheric atomic hydrogen. DE 1 observations on 14 October 1981 were compared with a spherically symmetric Chamberlain (1963) model of the atomic hydrogen distribution, after the application of the radiative transfer equations. The study showed that the optimum fit for data used a Chamberlain's classical model of exobase temperature $T=1050 \mathrm{~K}$ and exobase density $n_{\mathrm{c}}=4.4 \times 10^{4} \mathrm{~cm}^{-3}$ at $r_{\mathrm{c}}=1.08 R_{\mathrm{E}}$. This model fitting was still representative of the neutral hydrogen atom density distribution during the whole satellite operating period. Thus, we choose to compare the following exospheric model results to this baseline.

\subsection{Exospheric models in use}

\subsubsection{Description}

We choose to test models from the Mass-SpectrometerIncoherent-Scatter (MSIS) family (MSIS-86, MSISE-90 and NRLMSISE-00 models) and the exospheric $\mathrm{H}$ model from Hodges Jr. (1994).

The Mass-Spectrometer-Incoherent-Scatter models provide the temperature and the density of the following neutral 
chemical species - He, O, $\mathrm{N}^{2}, \mathrm{O}^{2}, \mathrm{Ar}, \mathrm{H}$, and $\mathrm{N}-$ in the upper atmosphere (altitude above $20 \mathrm{~km}$ ). These models stand on several data sources like rockets, satellites (OGO 6, San Marco 3, AEROS-A, AE-C, AE-D, AE-E, ESRO 4 and DE 2) and incoherent scatter radar (Millstone Hill, St. Santin, Arecibo, Jicamarca and Malvern) measurements. The first model of this kind, MSIS-86, was built thanks to the analysis work and a compilation of these data by A. E. Hedin and his team. Note that the MSIS- 86 model constitutes the upper part of the COSPAR International Reference Atmosphere (CIRA, 1986). The utilization of these MSIS models needs the prior knowledge of the temporal and spatial parameters (year, day, hour and altitude, latitude, longitude), the solar radio flux F10.7 and the magnetic index Ap input.

The MSISE-90 version model (Hedin, 1991) is appropriate for studies below $120 \mathrm{~km}$ of altitude. Under $72.5 \mathrm{~km}$, this model is primarily based on the MAP Handbook (Labitzke et al., 1985) with additional data from the National Meteorological Centre below $20 \mathrm{~km}$. Above $72.5 \mathrm{~km}$, we simply get a revised version of the MSIS- 86 model.

Otherwise the NRLMSIS-00 model (Picone et al., 2002) is also a revised version, of MSISE-90 model this time, developed by M. Picone, A. Hedin and D. Drob. The main differences, compared to the previous version, are the integration of an additional dataset and the possibility to take oxygen ionic species into account at altitudes above $500 \mathrm{~km}$.

As these models are limited to an altitude of $3000 \mathrm{~km}$ and in order to quantify hydrogen density in the global inner magnetosphere, we need to extrapolate these models further in space. Above $500 \mathrm{~km}$, we assess the hydrogen density distribution by the following extrapolation, assuming a hydrostatic equilibrium:

$n_{\mathrm{H}}=n_{H 500 \mathrm{~km}} \exp \left(\frac{m_{\mathrm{H}} g_{0} R_{\mathrm{E}} \times 10^{3}}{k_{\mathrm{B}}} \frac{\frac{500}{R_{\mathrm{E}}+500}-\frac{z}{R_{\mathrm{E}}+z}}{T_{\text {exosphere } 500 \mathrm{~km}}}\right)$

with $m_{\mathrm{H}}$ the mass of a hydrogen atom $[\mathrm{kg}], g_{0}$ the gravity acceleration $\left[\mathrm{m} \mathrm{s}^{-2}\right], R_{\mathrm{E}}$ the Earth's radius $[\mathrm{km}], k_{\mathrm{B}}$ the Boltzmann constant $\left(1.3806 \times 10^{-23} \mathrm{~J} \mathrm{~K}^{-1}\right), z$ the altitude $[\mathrm{km}], n_{H 500 \mathrm{~km}}$ the hydrogen density at $z=500 \mathrm{~km}\left[\mathrm{~cm}^{-3}\right]$ and $T_{\text {exosphere }} 500 \mathrm{~km}$ the exospheric temperature at $z=500 \mathrm{~km}$ $[\mathrm{K}]$. The use of this extrapolation was checked for the validity of MSIS models (i.e., $3000 \mathrm{~km}$ ).

Concerning the exospheric $\mathrm{H}$ model from Hodges (1994), a third-order spherical harmonic expansion in longitude and colatitude is used to represent hydrogen density at a particular radius. The corresponding harmonic expansion coefficients were originally derived from a Monte Carlo simulation of the terrestrial hydrogen exosphere. This model provides neutral hydrogen atom density in the Earth's exosphere from a radial distance of $6640 \mathrm{~km}$ to $62126 \mathrm{~km}$, for both solstice and equinox conditions, and for the four following levels of solar activity: F10.7 $=[80 ; 130 ; 180 ; 230]$ Solar Flux Unit (S.F.U. $=10^{-22} \mathrm{~W} \mathrm{~m}^{-2} \mathrm{~Hz}^{-1}$ ). Coefficients are given in the form of tables for given altitude values.

\subsubsection{Model utilization impact on estimated neutral hydrogen atom density}

To get an idea of the exospheric hydrogen density, we begin with the extrapolation of MSIS models.

\section{MSIS models at high altitude}

We are reminded that MSIS models are extrapolated in the way mentioned in the previous section at altitudes above $500 \mathrm{~km}$. So in order to compare these models to the baseline we defined before, we choose the conditions of Rairden's measurements as input of our models. Simulations are computed for the 287th day of the year 1981, at a local time of $9.4 \mathrm{~h}$ and for a magnetic activity equivalent to an Ap index of 73 (the value on 14 October 1981 was found thanks to the National Geophysical Data Centre). Solar activity by the solar radio flux F10.7 parameter is settled to 256.2 S.F.U. for the previous day and 222.28 S.F.U. for the mean solar radio flux during the three previous months. These values were obtained thanks to the Space Weather Prediction Centre data service. Neutral atom hydrogen density results are shown in Fig. 6, red triangles correspond to Rairden's best-fit points. We can immediately note that the MSIS- 86 and MSISE-90 models give almost the same values of exospheric hydrogen density. Although the NRLMSISE-00 model is also extrapolated in the same way, it seems that this model version tends to moderately overestimate hydrogen density whatever the radial distance is, which is good at low altitudes but worsens when radial distance $r$ increases. To compare with the MSIS-86 model, the maximum density ratio we get is 1.24 at $r=1.08 R_{\mathrm{E}}$. Globally, recent versions of MSIS models do not bring so much interest at those altitudes: as the authors of these models recommend themselves to take the MSIS-86 version at altitudes above $120 \mathrm{~km}$ (more recent MSIS versions improve low-altitude prediction), we will focus on the MSIS-86 model in more detail in the next paragraph. A comparison with Rairden's baseline will be discussed later in the section.

\section{Time, solar and magnetic activity influence on the MSIS-86 model}

We aim at determining the influence of the physical parameters (date, local time, Ap index and solar radio flux F10.7) taken as input of the MSIS-86 model on exospheric hydrogen density distribution.

Firstly, we focus on seasonal and local time effects on exospheric neutral hydrogen atom density. Thus, calculations are done for equinox and solstice times, i.e., on the 80th, 172th, 266th and 356th day of the year, and the model is parameterized so that the magnetic and solar activity get average values, more precisely $\mathrm{Ap}=10[2 \mathrm{nT}]$ (for a quiet time) and the solar radio flux of the day before and its mean value over the three previous months are both taken to 120 S.F.U. 


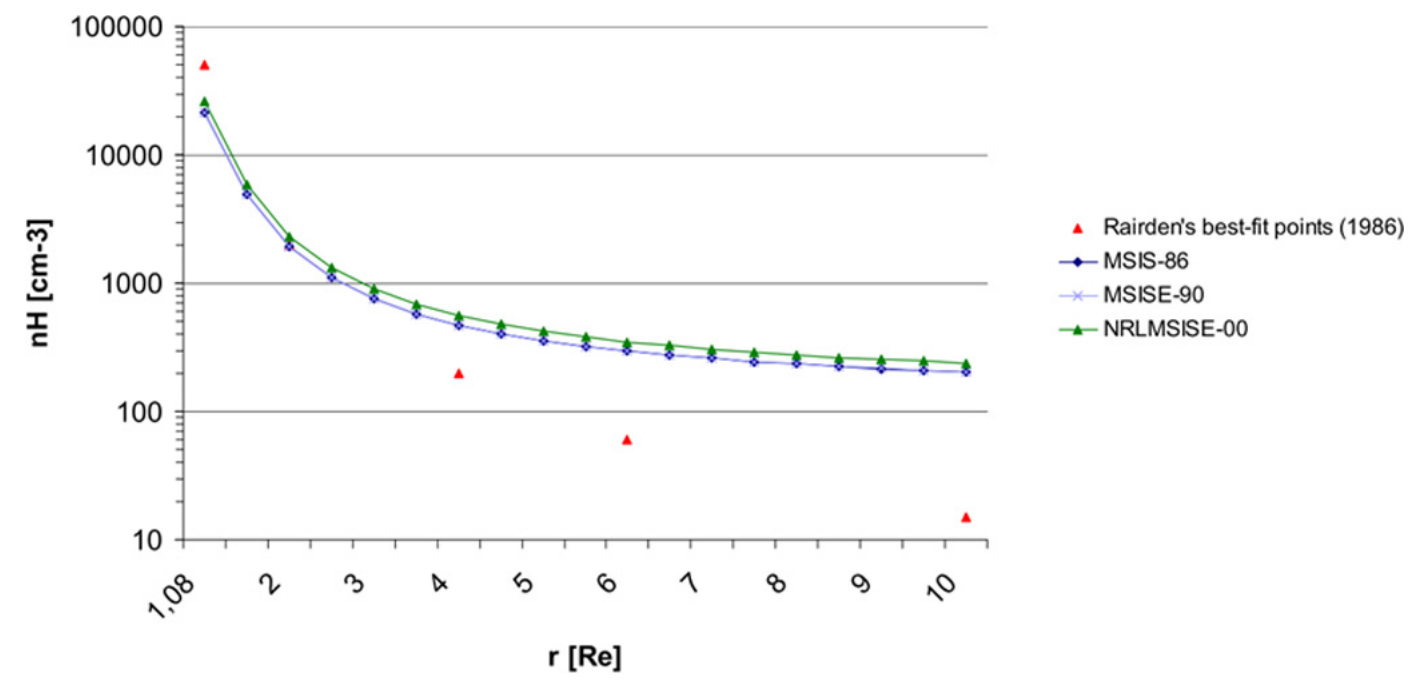

Fig. 6. Exospheric hydrogen density versus radial distance with extrapolated MSIS models.
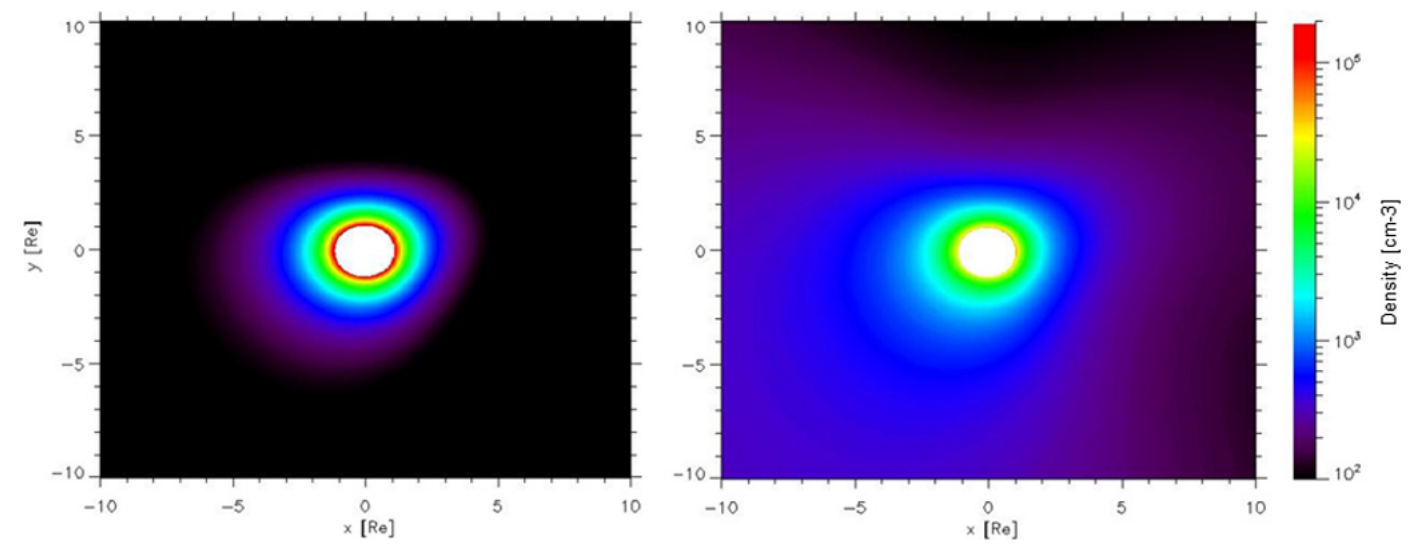

Fig. 7. Exospheric hydrogen density equatorial maps for a solar radio flux of 60 S.F.U. and 200 S.F.U. (respectively on left and right sides) with the MSIS-86 model.

The highest hydrogen densities obtained are respectively $1.73 \times 10^{5}, 1.80 \times 10^{5}, 1.61 \times 10^{5}$ and $1.77 \times 10^{5} \mathrm{~cm}^{-3}$ for spring and autumn equinoxes and summer and winter solstices, whatever the altitude is. We understand that the density values are higher in September than in June, as during the equinox time North and South Hemispheres are identically heated, contrary to the solstice time. So temperature is higher and consequently, it is natural to have higher hydrogen density. However, it is not possible to check this physical response with the winter solstice and spring equinox. Moreover, equinox times show unexpected differences with the maximum of hydrogen density. In testing exospheric hydrogen density distribution in local time, we choose to plot hydrogen density versus radial distance for 03:00, 09:00, 15:00 and 21:00 Local Time. It appears that hydrogen density is much more important at 15:00 LT for radial distances greater than $2 R_{\mathrm{E}}$. This is what we observe on the equatorial hydrogen density maps in Fig. 7 as a kind of exospheric growth at 15:00 LT. Atmospheric layers are the most heated at noon, but given the system response time, it is not surprising to have the maxima of density around 15:00 LT. The maximum density ratio we get between the graphs at 15:00 and 21:00 LT reaches 2.19, compared to 1.22 between the 09:00 and 21:00 LT graphs at $r=10 R_{\mathrm{E}}$. Comparing the density profile at 09:00, 03:00 and 21:00 LT, the first one reveals that hydrogen density is a bit greater than in the last two cases which are very similar, which can again be explained by the fact that of course the dayside is more heated than the nightside where the exosphere cannot be more dilated. If we assess the neutral hydrogen atom profiles at 15:00 LT for equinox and solstice times, we find a maximum density ratio of 1.14 between the spring equinox and the winter solstice at $r=10 R_{\mathrm{E}}$, so local time influence acts more on exospheric hydrogen density than seasonal influence.

Now, we focus on magnetic activity influence thanks to the Ap index parameter. Calculations in the equatorial 
plane are done for an Ap index of 0, 15, 50, 200 and 400 [2 $\mathrm{nT}]$ : the corresponding maxima found for hydrogen density are $1.94 \times 10^{5}, 1.76 \times 10^{5}, 1.65 \times 10^{5}, 1.33 \times 10^{5}$ and $9.95 \times 10^{4} \mathrm{~cm}^{-3}$. Hydrogen density maps for $\mathrm{Ap}=15$ and $\mathrm{Ap}=200[2 \mathrm{nT}]$ show that the exospheric halo expands as the magnetic activity increases: curiously the exosphere is more dilated around 03:00 LT this time, apparently due to particles from the plasma sheet that deposit their energy in this local time region during a short period of time, when they get inward and eventually precipitate. Thus, the external atmospheric layers are heated by this process and this is the reason why we may observe an expansion in this region. If we compare density profiles as a function of altitude for $\mathrm{Ap}=15$ and $\mathrm{Ap}=200[2 \mathrm{nT}]$ at 03:00 LT this time, the maximum density ratio we get is 2.25 at $r=10 \mathrm{Re}$, so magnetic activity is also more influent on the exospheric hydrogen density than seasonal effects.

The MSIS- 86 model needs, as input, the solar radio flux of the day before and its mean value over the three previous months. In our simulations, we decide to take both equal to 60, 120, 200 and 400 S.F.U. Results lead to hydrogen density maxima of $4.48 \times 10^{5}, 1.80 \times 10^{5}, 5.25 \times 10^{4}$ and $3.1 \times 10^{3} \mathrm{~cm}^{-3}$, respectively. Density maps for a solar radio flux F10.7 of 60 and 200 S.F.U. are illustrated in Fig. 7: we notice that the exospheric halo expands as solar radio flux increases. It seems that for strong values of the solar radio flux, the hydrogen density at low altitude is lower than for weaker solar radio flux cases, but then from $r=2 R_{\mathrm{E}}$, it is the opposite situation. This is confirmed by plotting the hydrogen density versus radial distance for F10.7 $=60$ and F10.7 $=200$ S.F.U. at 15:00 LT. At $r=5 R_{\mathrm{E}}$, the hydrogen density is 3.8 times stronger for a solar radio flux of 200 S.F.U. than for 60 S.F.U. At $r=10 R_{\mathrm{E}}$, this ratio reaches 5.8. This makes sense, considering that the solar radio flux is well correlated with the ultraviolet flux: as ultraviolet radiation heats the ionosphere and the atmosphere, if the solar radio flux undergoes substantial variations then substantial temperature variations are also expected, involving nonnegligible variations in the hydrogen density distribution. It appears that the solar radio flux is a key parameter for exospheric hydrogen density assessment and so for a further estimation of particle losses by charge exchange.

\section{Time, solar and magnetic activity influence on the exospheric $\mathbf{H}$ model from Hodges}

As for the MSIS-86 model, we aim at determining the influence of time, magnetic and solar activity on the distribution profile of hydrogen density with the Hodges exospheric $\mathrm{H}$ model. This last model is not as easily parameterizable as the MSIS-86 model: although local time and latitude localisations are free, we can only select an equinox or a solstice time, for a solar radio flux of $80,130,180$ or 230 S.F.U. The exospheric hydrogen density profiles with these eight datasets are illustrated in Fig. 8 in the equatorial plane and at midnight. Whatever the period of the year, we notice that the lower the solar radio flux is, the greater the hydrogen density. The same observation was previously made with the MSIS86 model, but for radial distances lower than $2 R_{\mathrm{E}}$. The maximum density ratio, obtained for a solstice time between the 80 S.F.U. and 230 S.F.U. curves, is 7.02 at $r=1.04 R_{\mathrm{E}}$. Considering the time influence, the period of the year does not impact so much on hydrogen density distribution, although density values are always weaker during equinoxes than during solstices (the maximum density ratio is equal to 1.33 for a solar radio flux of 180 S.F.U. at $r=1.42 R_{\mathrm{E}}$ ). Moreover, this density difference tends to decrease when the solar radio flux is stronger. The hydrogen density maps with the Hodges model were set by a power law interpolation and can be found in Fig. 9 for the extreme values of the solar radio flux (80 and 230 S.F.U.), both for solstice and equinox times. Clear observation of solar activity influence on hydrogen density is only visible well during solstices: again, we can note the density increase for weaker values of flux. From the local time point of view, few variations are perceptible with these maps. Actually, in the most critical case for a solar radio flux of 80 S.F.U., we get a maximum density ratio of 2.73 between the 03:00 and the 15:00 LT curves at the lowest altitude point. However, between $2 R_{\mathrm{E}}$ and $6 R_{\mathrm{E}}$, density ratio is weak $(<1.5)$, so local time has only a small influence on the hydrogen density distribution in the inner magnetosphere.

\section{Discussion on the MSIS-86 and the exospheric $\mathrm{H}$ model utilization}

A comparison of hydrogen density evolution according to the altitude, with the exospheric $\mathrm{H}$ model from Hodges and the MSIS- 86 model, is illustrated in Fig. 10 with regards to Chamberlain's best-fit measurements taken by DE 1 on 14 October 1981 (Rairden et al., 1986). Calculations were done under the measurement conditions, more specifically with an Ap index of 73, a solar radio flux of 256.2 S.F.U. on 13 October 1981 and 222.28 S.F.U. for the mean flux value of the three previous months, and at 09:24LT. In the case of the Hodges exospheric H model, we had to choose the closest conditions (F10.7 $=230$ S.F.U. at equinox time). Despite the fact that these are approximate conditions, we can immediately see that this model is closer to Rairden's observations. Concerning the MSIS-86 model, we can easily get an order of magnitude in density between Rairden's best-fit points (derived from the fitting of DE- 1 Lyman- $\alpha$ observations) and the model profile for given radial distance values: this discrepancy can be well understood by the fact that the MSIS-86 model is extrapolated over $500 \mathrm{~km}$ of altitude, so the extrapolation is getting worse and worse as the altitude increases. Under $r=2 R_{\mathrm{E}}$, the MSIS- 86 model underestimates hydrogen density by roughly a factor 2 at the most and over this radial distance, the model gives increasingly worse overestimation when the altitude increases, because of 


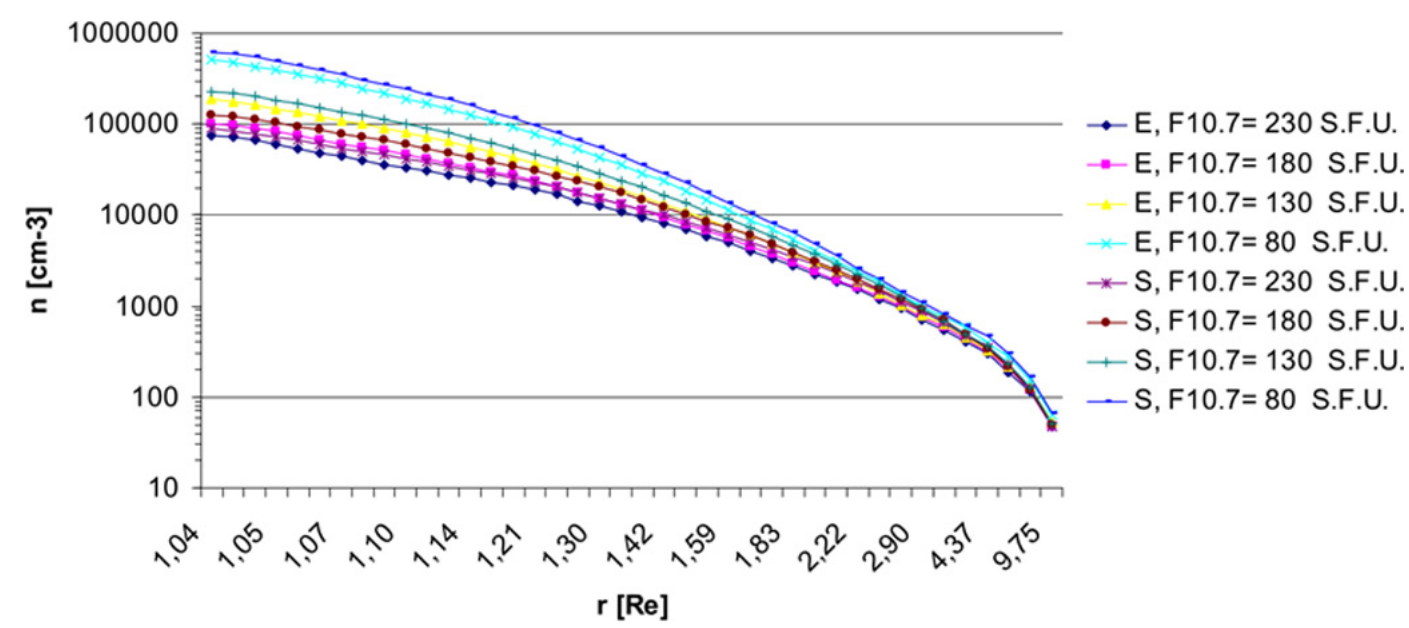

Fig. 8. Hydrogen density versus radial distance and radio solar flux at equinox (E) and solstice (S) at midnight with the exospheric $H$ model from Hodges.
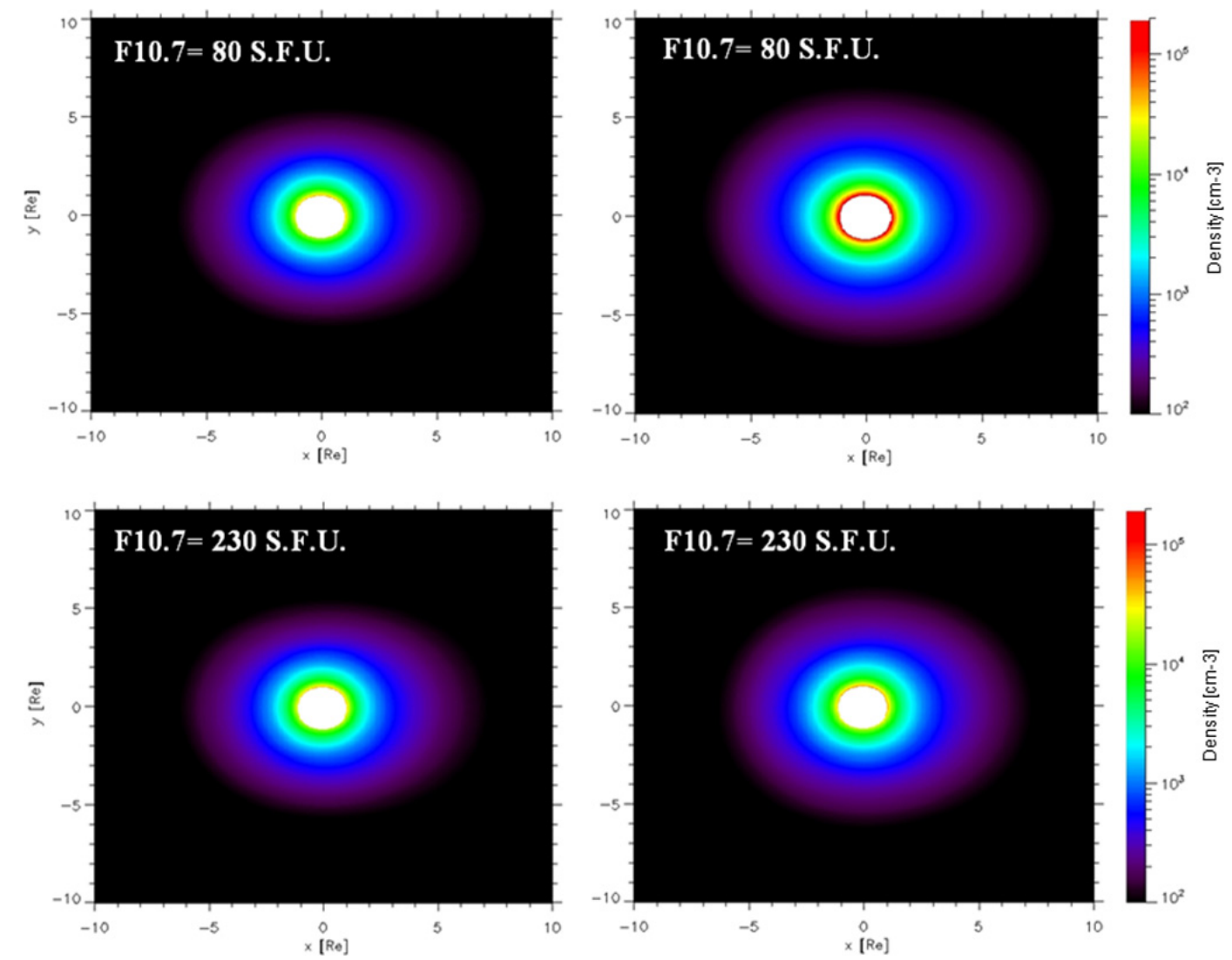

Fig. 9. Hydrogen density map at equinox (left panel) and solstice (right panel) for a solar radio flux of 80 S.F.U. and 230 S.F.U. with the exospheric $\mathrm{H}$ model from Hodges.

its extrapolation. Globally, it can give an idea of neutral hydrogen atom density by a factor 2 in the radiation belt slot region, whereas the Hodges exospheric $\mathrm{H}$ model can overestimate it by a factor 1.5 in this region and by roughly a factor 2 at the most over $10 R_{\mathrm{E}}$, in spite of its fixed parameter Ap set to 15 .
To summarise, seasonal influence is weak for both models, particularly for the MSIS-86 model. This last model gets an interesting sensitivity to local time, contrary to the exospheric $\mathrm{H}$ model, which highlights the duality day/night (asymmetry of the exosphere). Magnetic activity by the Ap parameter has a non-negligible effect on hydrogen density 


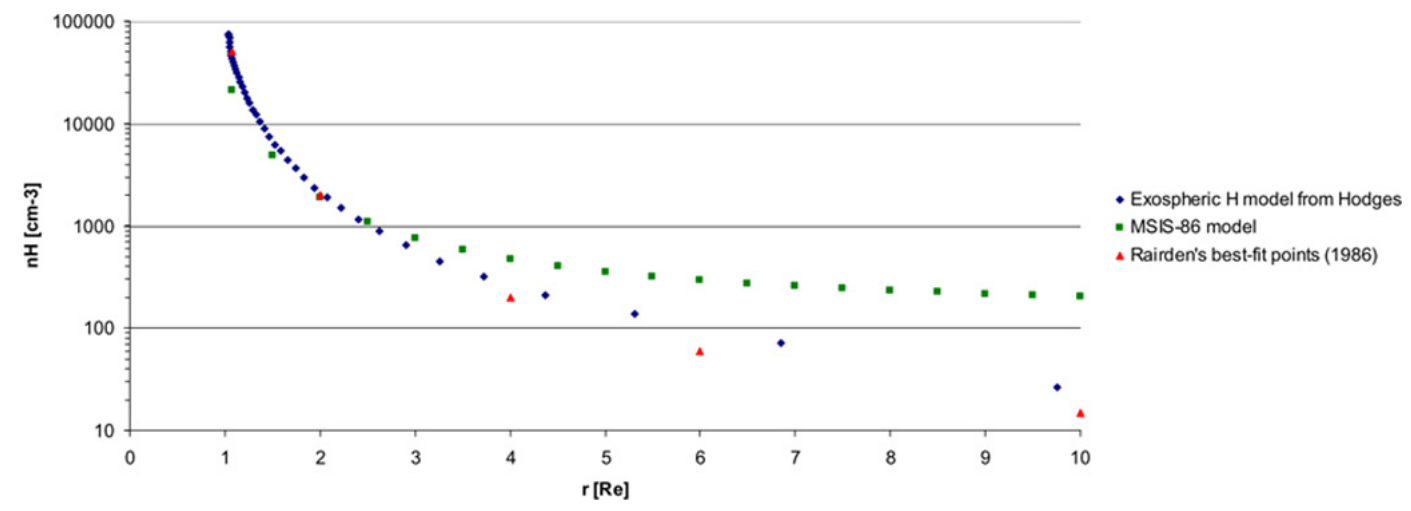

Fig. 10. Hydrogen density with the exospheric H model from Hodges and the MSIS-86 model compared to Rairden's best-fit points under the conditions of measurements made by DE 1 on 14 October 1981.
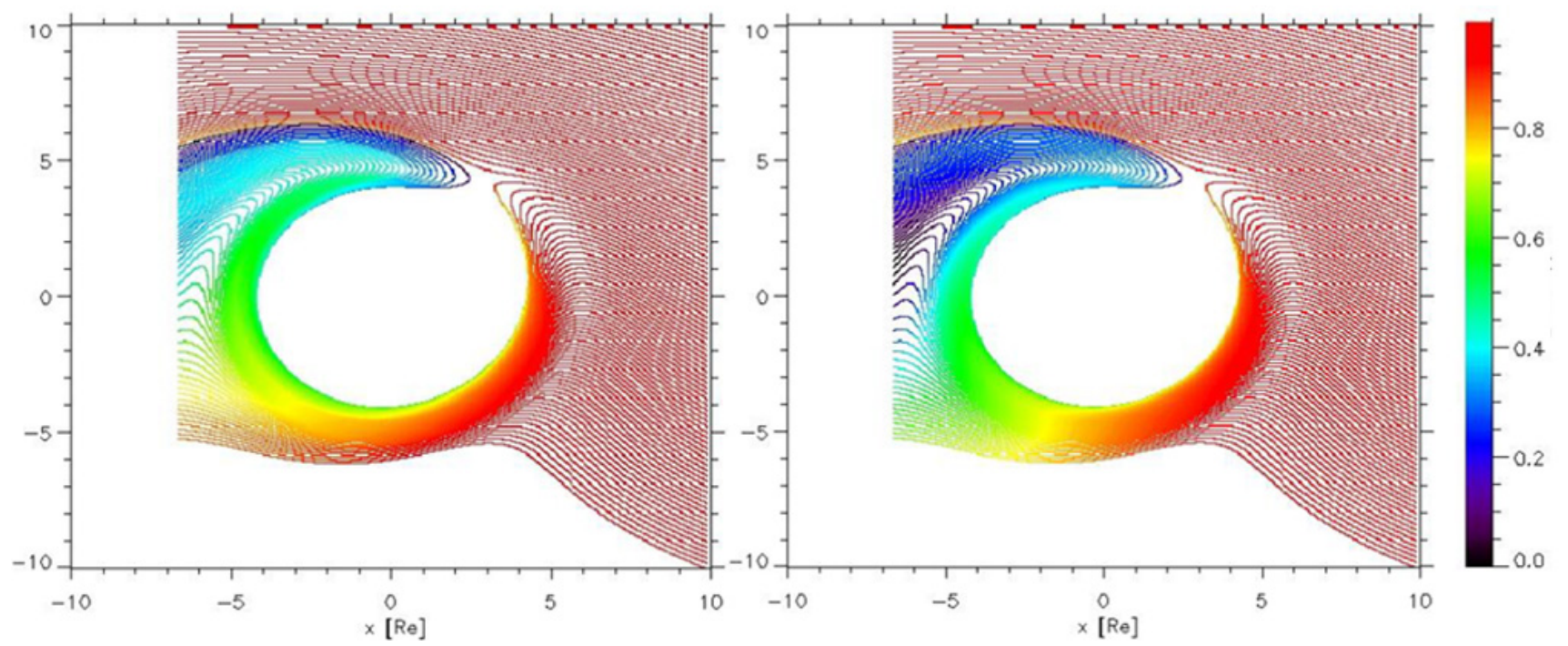

Fig. 11. Probability of remaining injected protons, along their trajectories, with the Hodges and the MSIS-86 exospheric models, (respectively, on left and right sides).

with the MSIS-86 model, but the exospheric $\mathrm{H}$ model from Hodges succeeds in giving a better density assessment without this index as input. In both models, the solar radio flux appears to be a key parameter for estimating exospheric hydrogen density and the advantage of the MSIS- 86 model is the possibility to parameterize this input precisely.

\subsection{A case example of proton loss}

An illustration of proton loss by charge exchange through exospheric atoms is available in Fig. 11. In this case, protons with $1 \mathrm{keV}$ energy are injected at $10 R_{\mathrm{E}}$ on the nightside and we basically assume, in a first approximation, that they are homogeneously spread all along the plasma sheet width. The Tsyganenko (1989) and Mcllwain models are selected for plasma transport modelling, and both MSIS-86 and Hodges exospheric models are used under the conditions de- scribed in Sect. 3.1. Then, we calculate the probability $p$ of the remaining protons along their trajectories by:

$p=\prod_{i=1}^{n}\left(1-\frac{\Delta t}{\tau_{i}}\right)$

with $1 / \tau_{i}$ the charge exchange frequency at the $i$-th time step $\Delta t\left(\Delta t \ll \tau_{i}\right)$, depending on the incident proton velocity, the charge exchange cross-section and the local hydrogen density given by an exospheric model. Time resolution is equal to $1 \mathrm{~s}$. The probability of collision between a proton and a neutral hydrogen atom is getting weaker and weaker as incident proton energy increases (particularly above few tens of $\mathrm{keV}$ ), due to the energy dependence of the charge exchange cross-section. Besides, it obviously decreases farther from the Earth and becomes almost stable down to $4 R_{\mathrm{E}}$.

Figure 11 shows in each case that proton losses mainly concern particles approaching closer to the Earth, which 
is quite understandable: the magnetic field magnitude is stronger near the Earth, so particles slow down in this region and consequently their residence time increases, as the probability of interaction with the exospheric atoms increases. It appears that losses mainly occur in the 03:00-15:00 LT sector, so globally on the dayside, which is reached by particles after a longer time. In addition to that, proton residence time is longer near the Earth partly due to the conflict between corotation and drift movements, which can result in almost "stagnant" protons. Apparently, exospheric model switch does not have so much influence, since differences from a model to another are small in the inner magnetosphere (see Fig. 10) where most of the charge exchange interactions happen: we can only notice an intensification of the losses in the 06:00-12:00 LT sector with the MSIS86 model, which is consistent with the previous observations as this model overestimates hydrogen density over an altitude of $1 R_{\mathrm{E}}$ compared to the exospheric $\mathrm{H}$ model from Hodges.

\section{Conclusion}

The aim of this qualitative study is to assess how model selection and the parameterisation impact on the global plasma transport modelling in the inner magnetosphere. Three dimensions of the modelling are essential: plasma source, transport and losses. The two last ones were studied here in more detail.

Concerning plasma transport, it was shown that basic magnetic field models (magnetic-activity, tilt and location dependent), such as Tsyganenko (1989), Mead and Fairfield, and Olson and Pfitzer quiet models, do not influence plasma dynamics as much as electric field models, which can lead to a very different final transport result. This was highlighted by the Maynard and Chen, Whipple and McIlwain model comparison, which leads to significant variations on the particle trajectory shape, energy evolution during transport and source location. None could have been identified as an "appropriate" electric field model for being used in such a modelling, but each of them presents interesting features: a Volland-Stern model type, like Maynard and Chen model, offers a good global description of plasma transport and source location in the plasma sheet; the shielding absence of the Whipple model appears to be an interesting characteristic with regards to Liemohn's work (2008); whereas the McIlwain model predicts well the plasmapause position and well describes the plasma dynamics inside the plasmasphere. So electric field model choice should be made according to the conditions in which it performs well, depending on the undertaken study.

Concerning losses while particles are transported, only proton losses by charge exchange were discussed here. For that, several exospheric models were tested with regards to Rairden's measurements. It appears that the exospheric $\mathrm{H}$ model from Hodges provides a better hydrogen density as- sessment, but the MSIS-86 model must be considered because of its easy parameterisation (accuracy on the solar radio flux input) and its sensitivity to local time. This comparison analysis should also be tested under different working conditions, but unfortunately only few data on hydrogen density measurement are available. Losses by the charge exchange phenomenon mainly concern protons up to a few tens of $\mathrm{keV}$ in the 03:00-15:00 LT sector near the Earth, where residence time is longer, so a good exospheric model is particularly needed in this region (below an altitude of $3 R_{\mathrm{E}}$ ).

Acknowledgements. We thank M. Liemohn (private communications) and P. Puhl-Quinn for her advice.

Topical Editor R. Nakamura thanks P. Puhl-Quinn and another anonymous referee for their help in evaluating this paper.

\section{References}

Alexeev, I. I., Belenkaya, E. S., and Clauer Jr., C. R.: A model of region 1 field-aligned currents dependent on ionospheric conductivity and solar wind parameters, J. Geophys. Res., 105(A9), 21119-21127, 2000.

Burke, W. J.: Penetration electric fields: A Volland Stern approach, J. Atmos. Solar-Terr. Phys., 69, 1114-1126, 2007.

Chamberlain, J. W.: Planetary coronae and atmospheric evaporation, Planet. Space Sci., 11, 901-960, 1963.

Christon, S. P., Williams, D. J., Mitchell, D. G., Huang, C. Y., and Franck, L. A.: Spectral Characteristics of Plasma Sheet Ion and Electron Populations During Disturbed Geomagnetic Conditions, J. Geophys. Res., 96(A1), 1-22, 1991.

CIRA: Part I: Thermosphere Model, edited by: Rees, D., Adv. Space Res., 8, \#5-\#6, 1986.

Dandouras, I., Cao, J., and Vallat, C.: Energetic ion dynamics of the inner magnetosphere revealed in coordinated ClusterDouble Star observations, J. Geophys. Res., 114, A01S90, doi:10.1029/2007JA012757, 2009.

Ebihara, Y. and Ejiri, M.: Simulation study on fundamental properties of the storm-time ring current, J. Geophys. Res., 105(A7), 15843-15859, 2000.

Fok, M.-C. and Moore, T. E.: Ring current modelling in a realistic magnetic field configuration, Geophys. Res. Lett., 24, 1775$1778,1997$.

Fok, M.-C., Moore, T. E., Kozyra, J. U., Ho, G. C., and Hamilton, D. C.: Three-dimensional ring current decay model, J. Geophys. Res., 100, 9619-9632, doi:10.1029/94JA03029, 1995.

Fok, M.-C., Wolf, R. A., Spiro, R. W., and Moore, T. E.: Comprehensive computational model of Earth's ring current, J. Geophys. Res., 106, 8417-8424, doi:10.1029/2000JA000235, 2001.

Harel, M., Wolf, R. A., Reiff, P. H., Spiro, R. W., Burke, W. J., Rich, F. J., and Smiddy, M.: Quantitative simulation of a magnetospheric substorm, 1, Model logic and overview, J. Geophys. Res., 86, 2217-2241, 1981.

Hedin, A. E.: Extension of the MSIS Thermospheric Model into the Middle and Lower Atmosphere, J. Geophys. Res., 96, 11591172, 1991.

Hodges Jr., R. R.: Monte Carlo simulation of the terrestrial hydrogen exosphere, J. Geophys. Res., 99(A12), 23229-23247, 1994. 
Huang, C.-L., Spence, H. E., Singer, H. J., and Tsyganenko, N. A.: A quantitative assessment of empirical magnetic field models at geosynchronous orbit during magnetic storms, J. Geophys. Res., 113, A04208, doi:10.1029/2007JA012623, 2008.

Korth, H., Thomsen, M. F., Borovsky J. E., and McComas, D. J.: Plasma sheet access to geosynchronous orbit, J. Geophys. Res., 104(A11), 25047-25061, doi:10.1029/1999JA900292, 1999.

Kovrazhkin, R. A., Sauvaud, J.-A., and Delcourt, D. C.: INTERBALL - Auroral observations of $0.1-12 \mathrm{keV}$ ion gaps in the diffuse auroral zone, Ann. Geophys., 17, 734-742, doi:10.1007/s00585-999-0734-9, 1999.

Labitzke, K., Barnett, J. J., and Edwards, B. (Eds.): Handbook MAP 16, SCOSTEP, University of Illinois, Urbana, 1985.

Liemohn, M. W.: Inner Magnetosphere Coupling (IMC) workshop, Finland, 28 July-1 August 2008.

Liemohn, M. W., Kozyra, J. U., Thomsen, M. F., Roeder, J. L., Lu, G., Borovsky, J. E., and Cayton, T. E.: Dominant role of the asymmetric ring current in producing the stormtime Dst*, J. Geophys. Res., 106(A6), 10883-10904, doi:10.1029/2000JA000326, 2001.

Maynard, N. C. and Chen, A. J.: Isolated cold plasma regions: Observations and their relation to possible production mechanisms, J. Geophys. Res., 80(7), 1009-1013, doi:10.1029/JA080i007p01009, 1975.

McCollough, J. P., Gannon, J. L., Baker, D. N., and Gehmeyr, M.: A statistical comparison of commonly used external magnetic field models, Space Weather, 6, S10001, doi:10.1029/2008SW000391, 2008.

McIlwain, C. E.: A Kp Dependent Equatorial Electric Field Model, Adv. Space Res., 6(3), 187-197, doi:10.1016/02731177(86)90331-5, 1986.

Mead, G. D. and Fairfield, D. H.: A Quantitative Magnetospheric Model Derived From Spacecraft Magnetometer Data, J. Geophys. Res., 80(4), 523-534, doi:10.1029/JA080i004p00523, 1975.

Olson, W. P. and Pfitzer, K. A.: Magnetospheric magnetic field modelling, Annual Scientific Report, AFOSR Contract No. F44620-75-C-0033, 1977.

Ostapenko, A. A. and Maltsev, Y. P.: Relation of the magnetic field in the magnetosphere to the geomagnetic and solar wind activity, J. Geophys. Res., 102(A8), 17467-17473, 1997.

Pfitzer, K. A., Olson, W. P., and Mogstad, T.: A time dependent source driven magnetospheric magnetic field model, EOS, 69, 426, 1988.

Picone, J. M., Hedin, A. E., Drob, D. P., and Aikin, A. C.: NRLMSISE-00 empirical model of the atmosphere: Statistical comparisons and scientific issues, J. Geophys. Res., 107(A12), 1468, doi:10.1029/2002JA009430, 2002.

Pierrard, V., Khazanov, G. V., Cabrera, J., and Lemaire, J.: Influence of the convection electric field models on predicted plasmapause positions during magnetic storms, J. Geophys. Res., 113, A08212, doi:10.1029/2007JA012612, 2008

Puhl-Quinn, P. A., Matsui, H., Jordanova, V. K., Khotyaintsev, Y., and Lindqvist, P.-A.: An effort to derive an empirically based, inner-magnetospheric electric field model: Merging Cluster EDI and EFW data, J. Atmos. Solar-Terr. Phys., 70, 564-573, 2008.

Pulkkinen, T. I. and Tsyganenko, N. A.: Testing the accuracy of magnetospheric model field line mapping, J. Geophys. Res., 101(A12), 27431-27442, 1996.
Rairden, R. L., Frank, L. A., and Craven, J. D.: Geocoronal Imaging With Dynamics Explorer, J. Geophys. Res., 91(A12), 1361313630, 1986.

Reeves, G. D., Weiss, L. A., Thomsen, M. F., and McComas, D. J.: A quantitative test of different magnetic field models using conjunctions between DMSP and geosynchronous orbit, in Radiation Belts: Models and Standards, Geophys. Monogr. Ser., vol. 97, edited by: Lemaire, J. F., Heynderickx, D., and Baker, D. N., pp. 167-172, AGU, Washington, D.C., 1996.

Ridley, A. J. and Liemohn, M. W.: A model-derived storm time asymmetric ring current driven electric field description, J. Geophys. Res., 107(A8), 1151, doi:10.1029/2001JA000051, 2002.

Spence, H. E., Kivelson, M. G., and Walker, R. J.: Static magnetic field models consistent with nearly isotropic plasma pressure, Geophys. Res. Lett., 14(8), 872-875, 1987.

Stern, D. P.: The motion of a proton in the equatorial magnetosphere, J. Geophys. Res., 80, 595-599, doi:10.1029/JA080i004p00595, 1975.

Stern, D. P.: The art of mapping the magnetosphere, J. Geophys. Res., 99(A9), 17169-17198, 1994.

Thomsen, M. F., McComas, D. J., Reeves, G. D., and Weiss, L. A.: An observational test of the Tsyganenko (T89a) model of the magnetospheric field, J. Geophys. Res., 101(A11), 2482724836, 1996.

Thomsen, M. F., Korth, H., and Elphic, R. C.: Upper cutoff energy of the electron plasma sheet as a measure of magnetospheric convection strength, J. Geophys. Res., 107(A10), 1331, doi:10.1029/2001JA000148, 2002.

Tsyganenko, N. A.: Global quantitative models of the geomagnetic field in the cislunar magnetosphere for different disturbance levels, Planet. Space Sci., 35, 1347-1358, 1987.

Tsyganenko, N. A.: A Magnetospheric Magnetic Field Model with a Warped Tail Current Sheet, Planet. Space Sci., 37(1), 5-20, doi:10.1016/0032-0633(89)90066-4, 1989.

Tsyganenko, N. A.: Effects of the solar wind conditions on the global magnetospheric configuration as deduced from data-based field models, in: European Space Agency Publication, ESA SP389, pp. 181-185, 1996.

Tsyganenko, N. A.: A model of the near magnetosphere with a dawn-dusk asymmetry, 1, Mathematical Structure, J. Geophys. Res., 107(A8), 1179, doi:10.1029/2001JA000219, 2002a.

Tsyganenko, N. A.: A model of the near magnetosphere with a dawn-dusk asymmetry, 2, Parameterization and fitting to observations, J. Geophys. Res., 107(A8), 1176, doi:10.1029/2001JA000220, 2002b.

Tsyganenko, N. A. and Mukai, T.: Tail plasma sheet models derived from Geotail particle data, J. Geophys. Res., 108(A3), 1136, doi:10.1029/2002JA009707, 2003.

Tsyganenko, N. A. and Sitnov, M. I.: Modeling the dynamics of the inner magnetosphere during strong geomagnetic storms, J. Geophys. Res., 110, A03208, doi:10.1029/2004JA010798, 2005.

Tsyganenko, N. A. and Usmanov, A. V.: Determination of the magnetospheric current system parameters and development of experimental geomagnetic field models based on data from IMP and HEOS satellites, Planet. Space Sci., 30(10), 985-998, 1982.

Tsyganenko, N. A., Singer, H. J., and Kasper, J. C.: Storm-time distortion of the inner magnetosphere: How severe can it get?, J. Geophys. Res., 108(A5), 1209, doi:10.1029/2002JA009808, 2003. 
Volland, H.: A semiempirical model of large-scale magnetospheric electric fields, J. Geophys. Res., 78(1), 171-180, doi:10.1029/JA078i001p00171, 1973.

Walker, R. J.: An evaluation of recent quantitative magnetospheric magnetic field models, Rev. Geophys., 14(3), 411-427, 1976.

Whipple Jr., E. C.: (U,B,K) Coordinates : A Natural System for Studying Magnetospheric Convection, J. Geophys. Res., 83(A9), 4318-4326, 1978.
Wygant, J. R., Rowland, D., Singer, H. J., Temerin, M., Mozer, F., and Hudson, M. K.: Experimental evidence on the role of the large spatial scale electric field in creating the ring current, J. Geophys. Res., 103(A12), 29527-29544, doi:10.1029/98JA01436, 1998. 